\title{
Handheld swept-source optical coherence tomography with angiography in awake premature neonates
}

\author{
Yasman Moshiri ${ }^{1}$, Alex T. Legocki ${ }^{1}$, Kanheng Zhou $^{2}$, Michelle T. Cabrera ${ }^{1,3}$, Kasra A. Rezaei ${ }^{1}$, \\ Kristina Tarczy-Hornoch ${ }^{1}$, Ruikang K. Wang ${ }^{1,2}$ \\ ${ }^{1}$ Department of Ophthalmology, ${ }^{2}$ Department of Bioengineering, University of Washington, Seattle, WA 98195, USA; ${ }^{3}$ Department of \\ Ophthalmology, Seattle Children's Hospital, Seattle, WA 98105, USA \\ Correspondence to: Ruikang K. Wang, PhD. Department of Bioengineering, University of Washington, Seattle, WA 98195, USA. Email: wangrk@uw.edu.
}

Background: Retinopathy of prematurity (ROP) can lead to retinal detachment and severe vision loss and is a common cause of childhood blindness. Optical coherence tomography angiography (OCTA) is a noninvasive imaging modality that can be used to detect potential abnormalities in the microvasculature in this population. The objective of this study is to assess the feasibility of a newly developed handheld swept source OCT (SS-OCT) device to successfully acquire structural vitreoretinal and retinal microvascular images in awake premature infants.

Methods: OCT and OCTA images were acquired at the time of routine ROP examinations from awake, unsedated preterm infants in the Neonatal Intensive Care Unit using a clinical research prototype handheld probe integrated with an SS-OCT system working at 1,060 nm wavelength and an imaging speed of 200,000 A-scans per second $(200 \mathrm{kHz})$, enabling volume OCT and OCTA scans. Each volume was acquired with approximately $36^{\circ}$ field of view $(\sim 6.3 \times 6.3 \mathrm{~mm}$ in infants $)$ in $4.8 \mathrm{~s}$. Quality of acquired OCT and OCTA volume images, microvascular information, and vitreoretinal features were determined by 3 -masked grader consensus.

Results: Twelve infants ( 5 females, mean gestational age 28.3 weeks, median birth weight $901 \mathrm{~g}$, stages 0 to 3 ROP) underwent a total of 73 individual eye imaging sessions. High-quality OCT images of the fovea and the optic nerve were present in 69/73 (94.5\%) and 56/73 (76.7\%) scans, respectively. Vitreous bands were observed in 10/73 (13.7\%); punctate hyperreflective vitreous opacities in 47/73 (64.4\%); epiretinal membrane (ERM) in 6/73 (8.2\%); and cystoid macular edema (CME) in 12/73 (16.4\%) scans. Mild vessel elevation was noted in $3 / 73(4.1 \%)$ images, and severe vessel elevation in 4/73 (5.5\%) scans. OCTA images obtained in 8 awake infants revealed good quality images of the foveal microvasculature in 11/19 (58\%) eye imaging sessions for $6 / 8$ (75\%) infants; and peripapillary microvasculature in $14 / 19$ (74\%) eye imaging sessions for $5 / 8(63 \%)$ infants.

Conclusions: The SS-OCTA handheld device can capture important vitreoretinal characteristics such as peripapillary and foveal microvasculature, as well as hyperreflective punctate vitreous opacities and tractional vitreous bands, which may predict ROP severity. These images were captured in awake, premature infants without the use of direct ocular contact, an eyelid speculum, or sedation.

Keywords: Retinopathy of prematurity (ROP); swept source optical coherence tomography; optical coherence tomography angiography (OCTA); handheld probe

Submitted Aug 22, 2019. Accepted for publication Sep 02, 2019.

doi: 10.21037/qims.2019.09.01

View this article at: http://dx.doi.org/10.21037/qims.2019.09.01 


\section{Introduction}

Retinopathy of prematurity (ROP) is a common cause of blindness, which afflicts neonates both in the United States and throughout the world today. Visual loss secondary to ROP is on the rise. Approximately 32,000 patients with ROP go on to develop visual impairment and permanent vision loss each year (1). Advancements in technology and infrastructure have led to improved survival of premature infants, which increases survival in individuals at risk for developing ROP (1). ROP is a vasoproliferative disorder that occurs in preterm infants due to disruption of normal development of the retina and its vasculature. While ROP can spontaneously regress in many cases, it can also lead to severe vision impairment and blindness due to retinal detachment. Screening eye examinations are essential for the timely diagnosis of ROP to prevent vision threatening consequences. Indirect ophthalmoscopy by an experienced ophthalmologist continues to be the mainstay of ROP screening, although wide-field retinal fundus photography is an acceptable alternative (2).

Handheld spectral domain optical coherence tomography (SD-OCT), a non-invasive imaging modality capable of capturing cross-sectional high resolution images, allows for detailed vitreoretinal structural imaging in premature infants $(3,4)$. Images obtained by SD-OCT from premature infants screened for ROP have demonstrated previously unrecognized structural features including cystoid macular edema (CME), epiretinal membranes (ERM), blood vessel anomalies, punctate hyperreflective vitreous opacities, vitreous bands, and retinoschisis $(4,5)$. While this device provides opportunities to better understand ROP pathogenesis and visual impairment in this population, its applicability to clinical ROP diagnosis remains limited, in part due to inadequate peripheral and vascular imaging, and difficulty in obtaining 3-dimensional (3D) scans. Furthermore, the only commercially available handheld SD-OCT device with FDA approval for infant use (Leica Microsystems, Buffalo Grove, IL, USA) can be challenging to use successfully in awake infants due to its slow imaging speed $(27 \mathrm{kHz})$ and short ranging distance $(\sim 3 \mathrm{~mm}$ in air), which makes finding the anatomic region of interest difficult and time consuming $(4,5)$.

We have developed a prototype handheld OCT with a $200 \mathrm{kHz}$ sweeping laser and a ranging distance of $12 \mathrm{~mm}$ (6). The prototype probe includes novel operatorassistance features such as a direct-view iris camera to more quickly center the probe, on-probe OCT display to maintain operator attention on the infant, and animated fixation targets in the probe to attract the infant's attention. This prototype also has high-speed OCTA angiography (OCTA) functionality. OCTA, a recent development in OCT imaging, allows for detailed quantification of the retinal vasculature without intravenous injections (7-10). A retinal vascular proliferative process is likely detectable by OCTA, and so analysis of OCTA images in infants with ROP may contribute to our understanding and diagnosis of this disease.

In this study, we present an investigational, handheld swept-source (SS) OCT device with angiographic (SSOCTA) (6) capability used to obtain high-quality structural vitreoretinal imaging and retinal microvascular imaging in awake premature infants at risk for ROP.

\section{Methods}

This was a prospective, observational study carried out at the University of Washington Medical Center (UWMC) in Seattle, Washington, and was approved by its Institutional Review Board. Handheld SS-OCT/SS-OCTA images were obtained from inpatients at the time of routine ROP examinations in the Neonatal Intensive Care Unit (NICU). Written consent was obtained prior to image acquisition from the parents or guardians of all study participants. This study was performed in accordance with the tenets of the Declaration of Helsinki.

An investigational, miniature handheld SS-OCTA device (Figure 1) was used in this study (6). The device was designed for use in clinical settings for imaging young children and infants, providing rapid, non-invasive structural and angiographic imaging of retina and choroid. Briefly, this device contained a 1,051 nm swept-source laser capable of an imaging speed of 200,000 A-scans per second $(200 \mathrm{kHz})$ that enables acquisition of 3D OCT and OCTA images with a scan time of 0.8 and $3.2 \mathrm{~s}$, respectively. The scanning angle on pupil was up to $\pm 36^{\circ}$, approximately a $6.3 \times 6.3 \mathrm{~mm}^{2}$ scan pattern, covering the full perifoveal region. Compared to $3 \mathrm{~mm}$ for a typical SD-OCT, the device provided a ranging distance of $12 \mathrm{~mm}$ that facilitated imaging in awake infants by allowing the operators to identify regions of interest within an acceptable time frame (a few seconds). The handheld probe included a unique pupil-finding feature, known as an iris viewer, which allowed for rapid positioning and alignment of the OCT probe with the optical axis of the infant's eye (see the insert of Figure 1A). Additionally, a real-time high-definition screen was available on the probe. Since the screen is 

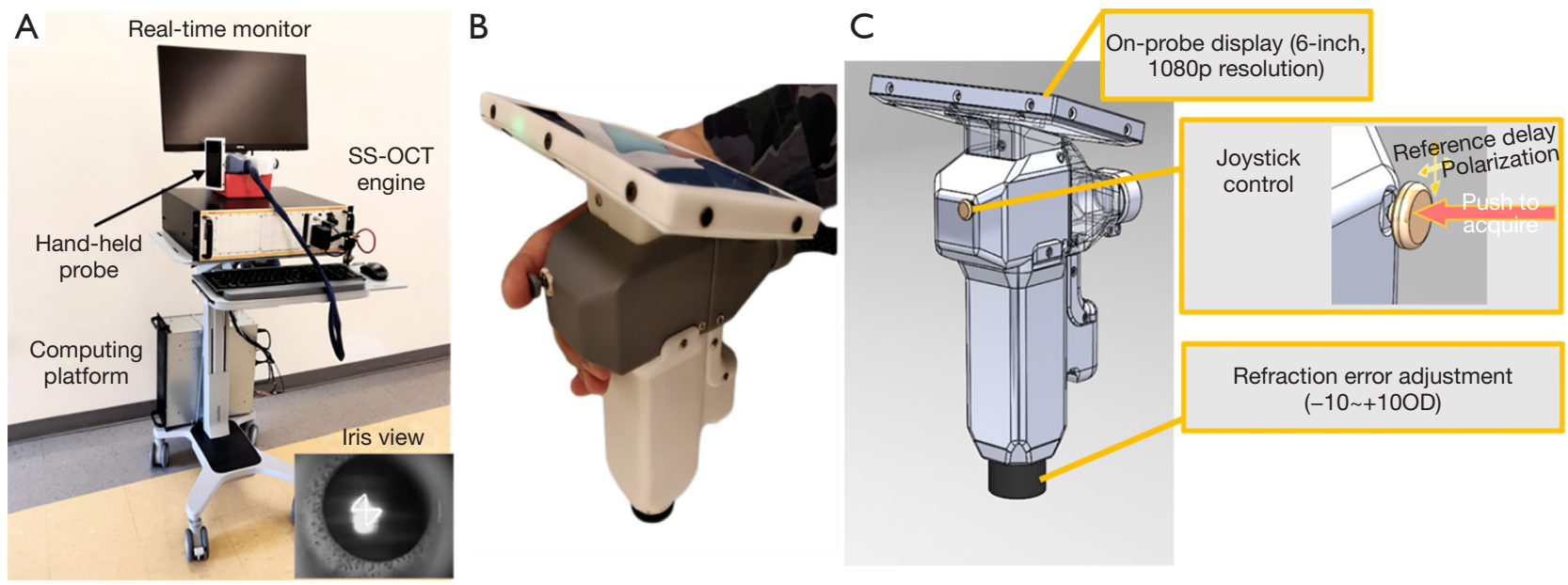

Figure 1 Swept source OCT system prototype designed for pediatric use, where built-in mini-screen on the handheld probe facilitates the imaging procedure. (A) Photograph of the full assembly of the system; insert is a typical screen shot from iris view during imaging operation; (B) photograph of the handheld probe; (C) simplified section view showing some functions on the probe that are adjustable by the operators during imaging. Unique features built into the probe include an iris view for rapid positioning and alignment of the OCT probe with the optic axis of the subject's eye.

mounted on the probe, the operator did not need to look away from the subject while positioning the probe and optimizing image quality, which improved infant safety and imaging efficiency. The total weight of the probe assembly was 948 grams. Finally, imaging was performed without the need for an eyelid speculum.

Study participants were infants undergoing routine ROP screening: infants less than 30 weeks gestational age, and/ or less than $1,500 \mathrm{~g}$ birth weight, or selected infants with a gestational age greater than 30 weeks with an unstable clinical course (at the discretion of the neonatologist). All participants underwent SS-OCT and SS-OCTA imaging following pharmacologic pupillary dilation on the same day as their routine ROP screening examinations using indirect ophthalmoscopy. The imaging schedule was dictated by the ROP screening examination schedule, which was determined by the treating ophthalmologist and based on standard screening criteria. Each premature infant underwent between 2 and 7 exams with SS-OCT and OCTA, 1-2 weeks apart. The ophthalmologists performing the eye examinations were masked to the results of the SSOCT and OCTA while determining ROP stage.

Images were obtained on awake, unsedated infants. No eyelid speculum was used. One to two drops of a mixture of $1 \%$ phenylephrine hydrochloride and $0.2 \%$ cyclopentolate ophthalmic solution (Cyclomydril, Alcon Laboratories, Geneva, Switzerland) were used to dilate the pupils before image acquisition. Four hundred B-scans (cross sectional OCT images) were acquired per volume (one scan across the eye). There were approximately two to five volumes taken per eye during each imaging session so as to maximize the quality of the foveal and optic nerve images taken. In some cases, an oral sucrose solution was administered to improve infant tolerance of the imaging. OCT images were generated using conventional OCT processing steps (11), and OCTA images were generated using a validated optical microangiography algorithm (12-14).

Two trained independent masked graders (YM and AL) viewed scans from all imaging dates for each infant. For each eye, the best quality OCT volumes including the optic nerve and foveal center were chosen and evaluated for presence or absence of several features of the retina and vitreous. Characteristics evaluated by the graders included punctate hyperreflective vitreous opacities, vitreous bands (tractional or non-tractional), ERM, CME, vessel elevation, scalloped retinal layers, hyporeflective vessels, and retinal spaces. A third trained, masked grader (MTC) reviewed all disagreements as the tie-breaker.

OCTA imaging was processed following usual convention, where image segmentation is performed on entire retinal volumes to separate the vascular networks into different layers (15). In this study, the retina was segmented into two layers: the superficial retina from ganglion cell layer (GCL) to inner plexiform layer (IPL), and the deep 

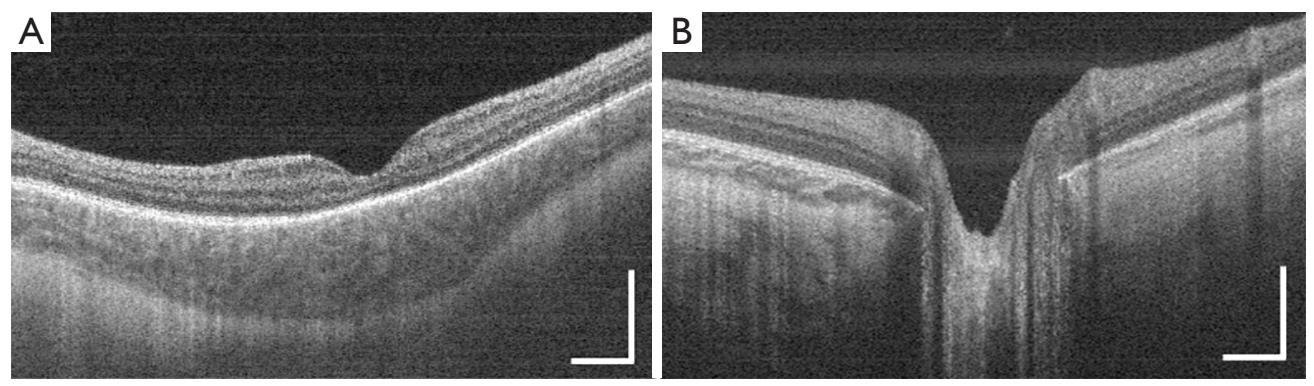

Figure 2 Typical examples of high-quality B-scan images extracted from 3D OCT scans obtained from (A) the fovea, and (B) the optic nerve. Note that the extracted images were single B-scans without averaging involved. The subject was an infant with gestational age $263 / 7$ weeks, stage 2 ROP, no plus disease and postmenstrual age 37 1/7 weeks at time of imaging. White scale bar =500 $\mu \mathrm{m}$. OCT, optical coherence tomography; ROP, retinopathy of prematurity.

retina from inner nuclear layer (INL) to external limiting membrane (ELM). Maximum intensity projection was provided for visualizing blood vessel networks in the en face view. False color maps of retinal vasculature were produced by coding superficial retinal vasculature as red and deep retinal vasculature as green to better visualize the depthresolved vasculatures (16).

As described by Maldonado et al., several specific OCT $\mathrm{B}$-scan vascular features are predictive of plus disease in ROP and are components of the Vascular Abnormality Score on OCT (VASO) (17). These include vessel elevation, scalloped retinal layers, hyporeflective vessels, and retinal spaces. Vessel elevation was defined as any spiked, pointed vessel easily distinguished from the normal smooth retinal surface found in subjects who did not have severe ROP. Scalloped retinal layers were defined as an inner plexiform layer or outer plexiform layer that appeared ruffled. Hyporeflective vessels were defined as vessels with dark lumina that cast shadow columns upon the underlying retinal layers. Retinal spaces were defined as the presence of hyporeflective spaces adjacent to vessels. In contrast to hyporeflective vessels, retinal spaces did not create shadowing and represented areas of acute angulation of vessels. All VASO findings were graded only if found $1 / 2$ disk diameter or further from the optic disk margin.

\section{Results}

\section{Subject characteristics}

There were twelve infants and a total of 73 individual eye examinations included in this study. Five infants were female $(41.7 \%)$ and seven were male $(58.3 \%)$. Infants had a mean birth weight of $901( \pm 304) \mathrm{g}$. Of the twelve infants included in the study, seven were white $(58.3 \%)$, two were Asian (16.7\%), two were Hispanic (16.7\%), and one was African American (8.3\%). Mean gestational age was 28.3 $( \pm 3.3)$ weeks. Mean postmenstrual age at the time of imaging was $36.5( \pm 3.2)$ weeks. Based on the clinical examination by indirect ophthalmoscopy, 7/12 (58\%) received a diagnosis of ROP at some point during the study. Of the 73 eye examinations corresponding to the imaging sessions from the 12 infants, 11 showed stage 1 ROP (15.1\%), 10 showed stage $2(13.7 \%)$, and 15 showed stage 3 (20.5\%). Neither plus nor pre-plus disease was observed among these infants.

\section{Vitreoretinal findings by handheld SS-OCT prototype}

Of the 73 volume scans obtained from the 12 infants, highquality images of the fovea (Figure $2 A$ ) and optic nerve (Figure 2B) were present in $69(94.5 \%)$ and $56(76.7 \%)$, respectively.

Vitreous bands (Figure 3A) were observed in 10/73 (13.7\%) of $3 \mathrm{D}$ scans. Of these, $9 / 10(90.0 \%)$ were tractional and $1 / 10$ (10.0\%) non-tractional. Punctate hyperreflective vitreous opacities (Figure 3B) were observed in 47/73 (64.4\%); ERM was seen in 6/73 (8.2\%); CME (Figure 3C) was present in $12 / 73$ (16.4\%). Mild vessel elevation was noted in $3 / 73$ (4.1\%) images, and severe vessel elevation in $4 / 73$ (5.5\%) scans (Figure 3D). Scalloped retinal layers, hyporeflective vessels, and retinal spaces were not observed in any images obtained from the twelve infants included in the study.

\section{Microvascular imaging by bandheld SS-OCTA}

A subset of 8 of our 12 study subjects underwent awake 

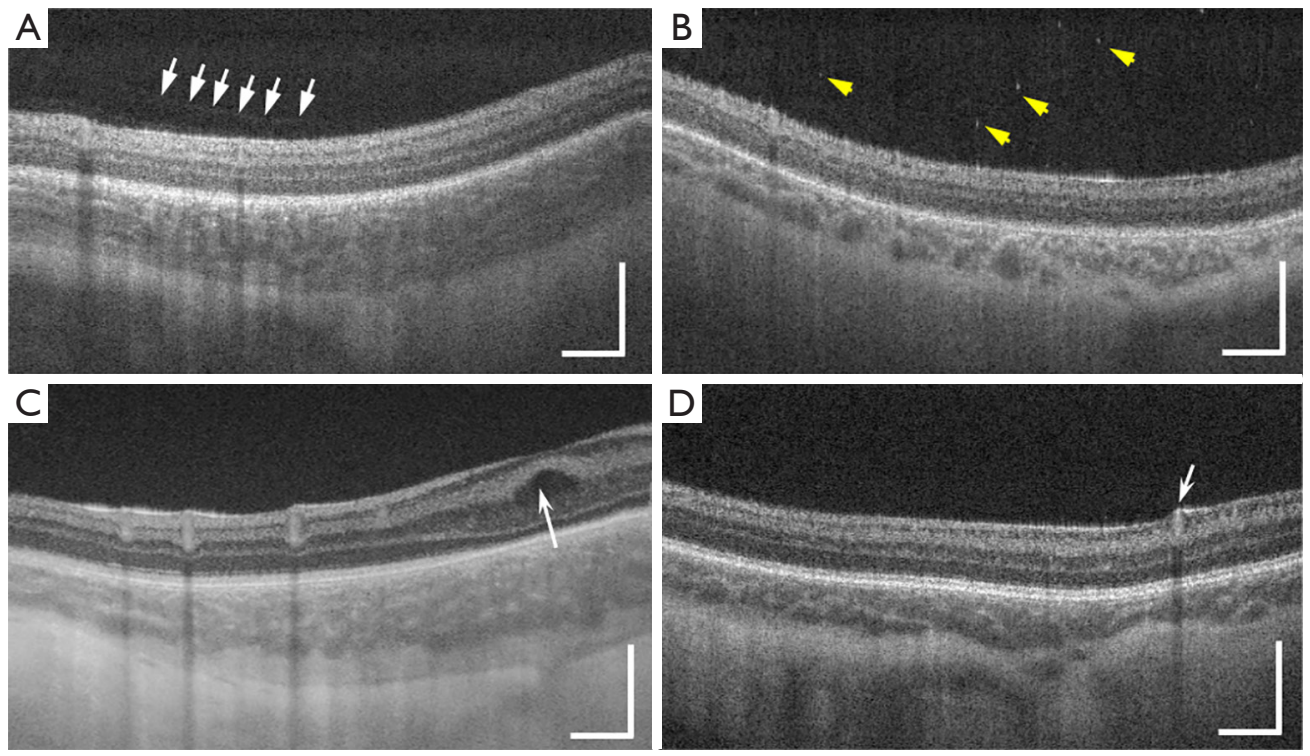

Figure 3 Vitreoretinal features in eyes with ROP observed by the handheld SS-OCT prototype system. (A) Vitreous bands (arrows) in an infant (left eye; stage 1, zone 2, no plus disease) with gestational age 26 6/7 weeks and postmenstrual age 33 4/7 weeks at the time of imaging. (B) Punctate hyperreflective vitreous opacities (arrows) in an infant (left eye; stage 0, zone 2, no plus disease) with gestational age 35 1/7 weeks and postmenstrual age 40 0/7 weeks at the time of imaging. (C) Cystoid macular edema (arrow) in an infant (left eye; stage 3 , zone 2, no plus disease) with gestational age $253 / 7$ weeks and postmenstrual age 39 6/7 weeks at the time of imaging. (D) Vessel elevation (arrow) in an infant (left eye; stage 3, zone 3, no plus disease) with gestational age 27 0/7 weeks and postmenstrual age 39 0/7 weeks at the time of imaging. Note that the images were single B-scans extracted from 3D scans, without averaging involved, except (C) was averaged from 5 repeated B-scans. White scale bar $=500 \mu \mathrm{m}$. ROP, retinopathy of prematurity; SS-OCT, swept source optical coherence tomography.

Table 1 Study participant demographics participating swept source optical coherence tomography (SS-OCTA) imaging

\begin{tabular}{lc}
\hline Total number of participants & Value \\
\hline Sex (male/female) & $5 / 3$ \\
Mean gestational age (weeks) & 27.3 (range, 26-35) \\
Mean birth weight (g) & 895 (range, 455-1,345) \\
Mean post-menstrual age at imaging & 39 (range, 31-44) \\
(weeks) & $0-3$ \\
Retinopathy of prematurity (ROP) staging & 19 \\
Imaging sessions & \\
\hline
\end{tabular}

retinal angiography using the handheld SS-OCTA prototype (see Table 1) with a wide-field scan pattern format of $6.3 \times 6.3 \mathrm{~mm}^{2}$. Good quality images were defined as those in which there was adequate quality to visualize the detailed microvasculature in at least one retinal layer without significant motion artifact or defocus. Good quality images of the foveal microvasculature were obtained in 11/19 (58\%) eye imaging sessions for $6 / 8$ (75\%) infants. Good quality images of peripapillary microvasculature were obtained in 14/19 (74\%) eye imaging sessions for 5/8 (63\%) infants.

Figure 4 provides typical examples of the OCTA images acquired from the deep and superficial retinal layers, together with the en face OCT images generated from all retinal layers. With this $6.3 \times 6.3 \mathrm{~mm}^{2}$ wide-field scan format, OCTA reveals the large retinal arteries and veins; however, capillaries are not as clearly delineated as in normal adult OCTA imaging (18-20). Nevertheless, the OCTA image may still provide valuable information regarding vessel area density, complexity, tortuosity, dilation and non-perfusion area.

\section{Discussion}

We have demonstrated that this novel handheld SS-OCT device with angiography capability can capture high-quality structural vitreoretinal images in 69/73 (94.5\%) imaging sessions of 12 awake, premature neonates. We were also able to obtain high-quality en face retinal angiographic 

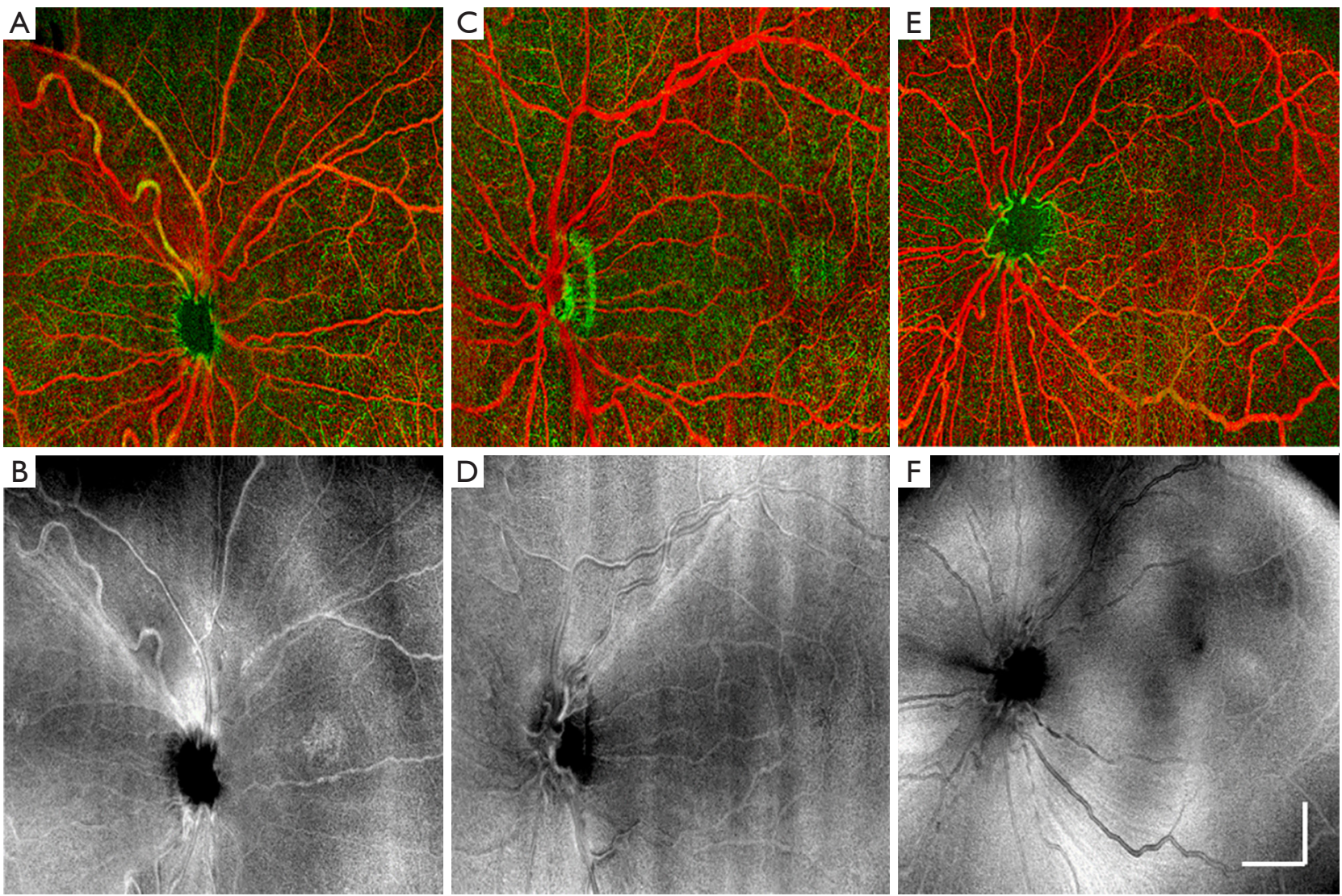

Figure 4 Examples of wide-field retinal angiograms $\left(\sim 6.3 \times 6.3 \mathrm{~mm}^{2}\right)$ acquired from the eyes of awake premature infants using handheld SSOCTA. The retinal angiograms are presented in color with superficial retinal vessels coded with red and deep retinal vessels coded with green. (A) Right eye of an infant at gestational age 27 0/7 weeks with stage 2, zone \#3 no plus disease, and postmenstrual age $441 / 7$ weeks at the time of imaging, and (B) corresponding OCT en face image generated from all retinal layers. (C) Left eye of an infant at gestational age 28 2/7 weeks with a mature eye, and postmenstrual age $432 / 7$ weeks at the time of imaging, and (D) corresponding OCT en face image generated from all retinal layers. (E) Left eye of an infant at gestational age $230 / 7$ weeks with stage 2, zone 2, no plus disease, and postmenstrual age 37 1/7 weeks at the time of imaging, and (F) corresponding OCT en face image generated from all retinal layers. White scale bar =1 mm. SS-OCTA, swept source optical coherence tomography angiography.

images of the macular microvasculature in $11 / 19(58 \%)$ imaging sessions of 6/8 (75\%) awake, premature neonates. While other investigational handheld OCT devices have demonstrated imaging in awake infants $(21,22)$, ours is the first to include a unique pupil-finding capability as well as a real-time on-probe monitoring screen.

This device also successfully captured high-quality images of key structures previously documented in premature infants using handheld SD-OCT imaging, including some associated with ROP severity. Known potential ROP severity biomarkers captured successfully by our prototype include punctate hyperreflective vitreous opacities and vitreous bands, both tractional and non- tractional (4). Additionally, this device demonstrated vascular features known to be associated with ROP severity, such as vessel elevation (17). Therefore, this device has the potential to provide insight into ROP severity that may ultimately inform management.

There are several advantages of this prototype over the currently available handheld SD-OCT, including greater imaging speed, greater usability of the handheld probe due to pupil and B-scan visualization, higher vitreous and choroidal detail, and angiography capability. We have demonstrated all of these functions in awake premature infants without the use of an eyelid speculum or ocular contact. Limitations of our study include small sample size 
and an infant population that lacked advanced ROP. Greater rates of advanced ROP may be associated with poorer imaging success due to greater media opacity, systemic illness and lower imaging tolerance.

In summary, we have presented a novel, handheld swept-source optical coherence tomography device with angiography capability that can capture important vitreoretinal features, some of which are associated with ROP severity. The device is user-friendly, well-tolerated in the premature infant population, and has the potential to identify important ROP pathophysiologic and diagnostic features in at-risk premature infants.

\section{Acknowledgments}

Funding: This study was supported in part by unrestricted grants from Research to Prevent Blindness and the NIH CORE Grant (EY001730) to the University of Washington Department of Ophthalmology, the Latham Foundation, the Washington Research Foundation in Seattle, the Knights Templar Eye Foundation, and the University of Washington Department of Bioengineering.

\section{Footnote}

Conflicts of Interest: Dr. Wang discloses intellectual property owned by the Oregon Health and Science University and the University of Washington. Dr. Wang also receives research support from Carl Zeiss Meditec Inc, Moptim Inc, Colgate Palmolive Company and Facebook Technology LLC. He is a consultant to Insight Photonic Solutions and Carl Zeiss Meditec. The other authors have no conflicts of interest to declare.

\section{References}

1. Blencowe H, Lawn JE, Vazquez T, Fielder A, Gilbert C. Preterm-associated visual impairment and estimates of retinopathy of prematurity at regional and global levels for 2010. Pediatr Res 2013;74 Suppl 1:35-49.

2. Yonekawa Y, Thomas BJ, Thanos A, Todorich B, Drenser KA, Trese MT, Capone A Jr. The cutting edge of retinopathy of prematurity care: Expanding the Boundaries of Diagnosis and Treatment. Retina 2017;37:2208-25.

3. Maldonado RS, Izatt JA, Sarin N, Wallace DK, Freedman S, Cotten CM, Toth CA. Optimizing Hand-held Spectral Domain Optical Coherence Tomography Imaging for Neonates, Infants, and Children. Invest Ophthalmol Vis
Sci 2010;51:2678-85.

4. Zepeda EM, Shariff A, Gillette TB, Grant L, Ding L, Tarczy-Hornoch K, Cabrera MT. Vitreous Bands Identified by Handheld Spectral-Domain Optical Coherence Tomography Among Premature Infants. JAMA Ophthalmol 2018;136:753-8.

5. Lee H, Proudlock FA, Gottlob I. Pediatric Optical Coherence Tomography in Clinical Practice-Recent Progress. Invest Ophthalmol Vis Sci 2016;57:OCT69-79.

6. Song S, Zhou K, Xu JJ, Zhang Q, Lyu S, Wang R. Development of a clinical prototype of a miniature handheld optical coherence tomography probe for prematurity and pediatric ophthalmic imaging. Biomed Opt Express 2019;10:2383-98.

7. Wang RK. Optical Microangiography: A Label Free 3D Imaging Technology to Visualize and Quantify Blood Circulations within Tissue Beds in vivo. IEEE J Sel Top Quantum Electron 2010;16:545-54.

8. Zhang A, Zhang Q, Chen CL, Wang RK. Methods and algorithms for optical coherence tomography-based angiography: a review and comparison. J Biomed Opt 2015;20:100901.

9. Chen CL, Wang RK. Optical coherence tomography based angiography. Biomed Opt Express 2017;8:1056-82.

10. Kashani AH, Chen CL, Gahm JK, Zheng F, Richter GM, Rosenfeld PJ, Shi Y, Wang RK. Optical coherence tomography angiography: A comprehensive review of current methods and clinical applications. Prog Retin Eye Res 2017;60:66-100.

11. Wang RK, Ma Z. A practical approach to eliminate autocorrelation artefacts for volume-rate spectral domain optical coherence tomography. Phys Med Biol 2006;51:3231-9.

12. Wang RK, An L, Francis P, Wilson DJ. Depth-resolved imaging of capillary networks in retina and choroid using ultrahigh sensitive optical microangiography. Opt Lett 2010;35:1467-9.

13. Yousefi S, Zhi Z, Wang RK. Eigendecomposition-based clutter filtering technique for optical microangiography. IEEE Trans Biomed Eng 2011;58:2316-23.

14. Zhang Q, Wang J, Wang RK. Highly efficient eigen decomposition based statistical optical microangiography. Quant Imaging Med Surg 2016;6:557-63.

15. Yin X, Chao JR, Wang RK. User-guided segmentation for volumetric retinal optical coherence tomography images. J Biomed Opt 2014;19:086020.

16. Zhang Q, Lee CS, Chao J, Chen CL, Zhang T, Sharma U, Zhang A, Liu J, Rezaei K, Pepple KL, Munsen R, Kinyoun 
J, Johnstone M, Van Gelder RN, Wang RK. Wide-field optical coherence tomography based microangiography for retinal imaging. Sci Rep 2016;6:22017.

17. Maldonado RS, Yuan E, Tran-Viet D, Rothman AL, Tong AY, Wallace DK, Freedman SF, Toth CA. Threedimensional assessment of vascular and perivascular characteristics in subjects with retinopathy of prematurity. Ophthalmology 2014;121:1289-96.

18. Bojikian KD, Chen CL, Wen JC, Zhang Q, Xin C, Gupta D, Mudumbai RC, Johnstone MA, Wang RK, Chen PP. Optic disc perfusion in primary open angle and normal tension glaucoma eyes using optical coherence tomography-based microangiography. PLoS One 2016;11:e154691.

19. Zhang Q, Huang Y, Zhang T, Kubach S, An L, Laron M, Sharma U, Wang RK. Wide-field imaging of retinal

Cite this article as: Moshiri Y, Legocki AT, Zhou K, Cabrera MT, Rezaei KA, Tarczy-Hornoch K, Wang RK. Handheld swept-source optical coherence tomography with angiography in awake premature neonates. Quant Imaging Med Surg 2019;9(9):1495-1502. doi: 10.21037/qims.2019.09.01 vasculature using optical coherence tomography-based microangiography provided by motion tracking. J Biomed Opt 2015;20:066008.

20. Zhang Q, Rezaei KA, Saraf SS, Chu Z, Wang F, Wang RK. Ultra-wide optical coherence tomography angiography in diabetic retinopathy. Quant Imaging Med Surg 2018;8:743-53.

21. Campbell JP, Nudleman E, Yang J, Tan O, Chan RVP, Chiang MF, Huang D, Liu G. Handheld Optical Coherence Tomography Angiography and Ultra-WideField Optical Coherence Tomography in Retinopathy of Prematurity. JAMA Ophthalmol 2017;135:977-81.

22. Viehland C, Chen X, Tran-Viet D, Jackson-Atogi M, Ortiz P, Waterman G, Vajzovic L, Toth CA, Izatt JA. Ergonomic handheld OCT angiography probe optimized for pediatric and supine imaging. Biomed Opt Express 2019;10:2623-38. 